\title{
Legendre Transform-Dual Solution for a Class of Investment and Consumption Problems with HARA Utility
}

\author{
Hao Chang ${ }^{1}$ and Xi-min Rong ${ }^{2}$ \\ ${ }^{1}$ School of Science, Tianjin Polytechnic University, Tianjin 300387, China \\ ${ }^{2}$ School of Science, Tianjin University, Tianjin 300072, China \\ Correspondence should be addressed to Hao Chang; ch8683897@126.com
}

Received 19 April 2014; Revised 17 May 2014; Accepted 18 May 2014; Published 28 May 2014

Academic Editor: Rajan Rakkiyappan

Copyright (c) $2014 \mathrm{H}$. Chang and X.-m. Rong. This is an open access article distributed under the Creative Commons Attribution License, which permits unrestricted use, distribution, and reproduction in any medium, provided the original work is properly cited.

\begin{abstract}
This paper provides a Legendre transform method to deal with a class of investment and consumption problems, whose objective function is to maximize the expected discount utility of intermediate consumption and terminal wealth in the finite horizon. Assume that risk preference of the investor is described by hyperbolic absolute risk aversion (HARA) utility function, which includes power utility, exponential utility, and logarithm utility as special cases. The optimal investment and consumption strategy for HARA utility is explicitly obtained by applying dynamic programming principle and Legendre transform technique. Some special cases are also discussed.
\end{abstract}

\section{Introduction}

The investment and consumption problem was originated from the seminal work of Merton [1,2], who first used stochastic optimal control to deal with a continuous-time portfolio selection problem with consumption behavior and obtained the closed-form solution of the optimal investment and consumption strategy under power utility and logarithm utility. In the following years, many scholars began to pay attention to the investment and consumption problems and obtained many research results. One can refer to the papers of Chacko and Viceira [3], Liu [4], Noh and Kim [5], Chang and Rong [6], and Chang et al. [7]. But these results were generally achieved under power utility, which was taken as a special case of hyperbolic absolute risk aversion (HARA) utility function.

HARA utility includes power utility, exponential utility, and logarithm utility as special cases. From the expression of HARA utility, we can see that HARA utility has more complicated structure than other utility functions, which leads to that there is a little work on portfolio selection problems with HARA utility in the existing literature. As a matter of fact, Grasselli [8] investigated a portfolio selection problem with HARA utility in the stochastic interest rate environment and verified that the optimal policy corresponding to HARA utility converges to the one corresponding to exponential utility and logarithm utility. Jung and Kim [9] provided a Legendre transform method to deal with an optimal portfolio model with HARA utility.

In recent years, Legendre transform is often used to deal with the complicated portfolio selection problems, for example, the optimal investment problems under the CEV model. One can refer to the work of Jonsson and Sircar [10], Xiao et al. [11], and Gao $[12,13]$. The advantage of Legendre transform is to transform the nonlinear $\mathrm{HJB}$ equation into a linear dual one and the structure of the solution under HARA utility is easy to conjecture. Therefore, it is very possible for us to use Legendre transform to obtain the optimal investment strategy of more complicated portfolio selection problems.

In this paper, we consider a class of investment and consumption problems, whose objective function is to maximize the expected discount utility of intermediate consumption and terminal wealth, and wish to obtain the optimal investment and consumption strategy for HARA utility. We provide a Legendre transform method to deal with this problem and obtain the explicit expression of the optimal investment and consumption strategy under HARA utility. Some special cases are also discussed. There are two main 
contributions in this paper. (i) We study a class of investment and consumption problems, whose objective function is more complicated than that of the optimal investment problems. In other words, we not only hope to obtain the optimal investment strategy, but also hope to obtain the optimal strategy of consumption. (ii) We obtain the explicit expression of the optimal investment and consumption strategy under HARA utility, which has rarely been studied in the optimal portfolio selection problems. It is all well known that most of portfolio selection problems are studied under power utility or exponential utility.

The structure of this paper is as follows. Section 2 formulates a class of investment and consumption problems with HARA utility, which wish to maximize the expected discount utility of intermediate consumption and terminal wealth. Section 3 uses Legendre transform to obtain the dual one of the HJB equation. Section 4 obtains the optimal investment and consumption strategy for HARA utility and Section 5 concludes the paper.

\section{The Model}

Throughout the paper, $(\cdot)^{\prime}$ represents the transpose of a matrix or sector, $\|x\|=\sqrt{x_{1}^{2}+\cdots+x_{n}^{2}}$ represents the norms of a sector $x=\left(x_{1}, x_{2}, \ldots, x_{n}\right)^{\prime}, \mathbb{E}(\cdot)$ represents the expectation, and $[0, T]$ represents the fixed and finite investment horizon. Assume that $\left(W_{1}(t), W_{2}(t), \ldots, W_{n}(t)\right)^{\prime}$ is a n-dimensional independent standard Brownian motion defined on complete probability space $\left(\Omega, \mathscr{F}, P,\left\{\mathscr{F}_{t}\right\}_{0 \leqslant t \leqslant T}\right)$, in which $\left\{\mathscr{F}_{t}\right\}_{0 \leqslant t \leqslant T}$ represents information flow generated by $\left(W_{1}(t), W_{2}(t), \ldots, W_{n}(t)\right)^{\prime}$.

Assume that the financial market is composed of $n+1$ assets, which are continuously traded on $[0, T]$. One is the risk-free asset (i.e., a bond), whose price at time $t$ is denoted by $P_{0}(t)$. Then $P_{0}(t)$ satisfies the following equation:

$$
d P_{0}(t)=r(t) P_{0}(t) d t, \quad P_{0}(0)=p_{0}>0
$$

where $r(t)>0$ is the risk-free interest rate.

The other $n$ assets are risky assets (i.e., stocks), whose prices at time $t$ are denoted by $P_{i}(t), i=1,2, \ldots, n$, and then $P_{i}(t)$ satisfies the following geometric Brownian motion (GBM):

$$
\begin{array}{r}
d P_{i}(t)=P_{i}(t)\left(b_{i}(t) d t+\sum_{j=1}^{n} \sigma_{i j}(t) d W_{j}(t)\right), \\
P_{i}(t)=p_{i}>0,
\end{array}
$$

where $b(t)=\left(b_{1}(t), b_{2}(t), \ldots, b_{n}(t)\right)^{\prime}$ and $\sigma(t)=\left(\sigma_{i j}(t)\right)_{n \times n}$ represent the appreciation rate sector and volatility matrix of the stocks, respectively, and $b_{i}(t)>r(t), i=1,2, \ldots, n$. In addition, we assume that $\sigma(t)=\left(\sigma_{i j}(t)\right)_{n \times n}$ satisfies the nondegenerated condition: $\sigma(t) \sigma^{\prime}(t)>0$, for all $t \in$ $[0, T]$, and $r(t), b(t)$, and $\sigma(t)$ are Borel-measurable bounded deterministic functions on $[0, T]$.
Assume that the amount at time $t$ invested in the $i$ th stock is denoted by $\pi_{i}(t), t \in[0, T]$. Letting $\pi(t)=$ $\left(\pi_{1}(t), \pi_{2}(t), \ldots, \pi_{2}(t)\right)^{\prime}, X(t)$ represents the wealth at time $t$ and $C(t)$ represents the consumption rate at time $t$; then, the amount invested in the bond is $\pi_{0}(t)=X(t)-\sum_{i=1}^{n} \pi_{i}(t)$. Suppose that the investor cannot consider transaction cost and short-sell the stocks; then, the wealth process $X(t)$ under trading strategy $(\pi(t), C(t))$ satisfies the following stochastic differential equation(SDE):

$$
\begin{aligned}
d X(t)= & \left(r(t) X(t)+B^{\prime}(t) \pi(t)-C(t)\right) d t \\
& +\pi^{\prime}(t) \sigma(t) d W(t), \quad X(t)=x_{0}>0,
\end{aligned}
$$

where

$$
B(t)=\left(b_{1}(t)-r(t), b_{2}(t)-r(t), \ldots, b_{n}(t)-r(t)\right)^{\prime} .
$$

Definition 1 (admissible strategy). An investment and consumption strategy $(\pi(t), C(t))$ is said to be admissible if the following conditions are satisfied:

(i) $\pi(t)$ and $C(t)$ are all $\mathscr{F}_{t}$-measurable and satisfy $\int_{0}^{T} C(t) d t<\infty$ and $\int_{0}^{T}\|\pi(t)\|^{2} d t<\infty ;$

(ii) $\mathbb{E}\left(\int_{0}^{T}\left\|\pi^{\prime}(t) \sigma(t)\right\|^{2} d t\right)<\infty$;

(iii) the $\operatorname{SDE}(3)$ has a pathwise unique solution according to $(\pi(t), C(t))$.

Assume that the set of all admissible investment and consumption strategies $(\pi(t), C(t))$ is denoted by $\Gamma=$ $\{(\pi(t), C(t)): 0 \leqslant t \leqslant T\}$. In this paper, we assume that the investor wishes to maximize the following objective function:

$$
\begin{aligned}
\underset{(\pi(t), C(t)) \in \Gamma}{\operatorname{Max}} \mathbb{E}\left(\int_{0}^{T} \alpha e^{-\beta t} U_{1}(C(t)) d t\right. & \\
& \left.+(1-\alpha) e^{-\beta T} U_{2}(X(T))\right),
\end{aligned}
$$

where $U_{1}(x)$ and $U_{2}(x)$ are all utility functions and $\beta>0$ is the subjective discount rate. The parameter $\alpha$ determines the relative importance of intermediate consumption and terminal wealth. When $\alpha=0$, expected utility only depends on terminal wealth and the problem (5) is reduced to an asset allocation problem.

In this paper, we choose hyperbolic absolute risk aversion (HARA) utility function for our analysis. HARA utility function with parameters $\eta, p$, and $q$ is given by

$$
\begin{array}{r}
U_{1}(x)=U_{2}(x)=U(\eta, p, q, x)=\frac{1-p}{q p}\left(\frac{q}{1-p} x+\eta\right)^{p}, \\
q>0, \quad p<1, \quad p \neq 0 .
\end{array}
$$

As a matter of fact, HARA utility function recovers power utility, exponential utility, and logarithm utility as special cases.

(i) If we choose $\eta=0$ and $q=1-p$, then we have

$$
U(0, p, 1-p, x)=\frac{x^{p}}{p}=U_{\text {power }}(x) \text {. }
$$


(ii) If we choose $\eta=1$ and $p \rightarrow-\infty$, then we have

$$
U(1, p, q, x)=\frac{-e^{-q x}}{q}=U_{\exp }(x) .
$$

(iii) If we choose $\eta=0, p \rightarrow 0$, and $q \rightarrow 1$, then we have

$$
U(0, p, q, x)=\ln x=U_{\log }(x) \text {. }
$$

\section{HJB Equation and Legendre Transform}

We define the value function $V(t, x)$ as

$$
\begin{aligned}
& V(t, x) \\
& =\sup _{(\pi(t), C(t)) \in \Gamma} \mathbb{E}\left(\int_{t}^{T} \alpha e^{-\beta t} U_{1}(C(t)) d t\right. \\
& \left.\quad+(1-\alpha) e^{-\beta T} U_{2}(X(T)) \mid X(t)=x\right),
\end{aligned}
$$

with boundary condition given by $V(T, x)=(1-\alpha) e^{-\beta T}$ $U_{2}(x)$.

Applying the principle of optimality (referring to Fleming and Soner [14]), if the value function $V(t, x) \in C^{1,2}([0, T] \times$ $\mathbb{R})$, then $V(t, x)$ satisfies the following Hamilton-JacobiBellman (HJB) equation:

$$
\sup _{(\pi(t), C(t)) \in \Gamma}\{\mathscr{A} V(t, x)\}=0
$$

where

$$
\begin{aligned}
\mathscr{A} V(t, x)= & V_{t}+r(t) x V_{x}+B^{\prime}(t) \pi(t) V_{x} \\
& +\frac{1}{2} \pi^{\prime}(t) \sigma(t) \sigma^{\prime}(t) \pi(t) V_{x x} \\
& -C(t) V_{x}+\alpha e^{-\beta t} U_{1}(C(t)),
\end{aligned}
$$

and $V_{t}, V_{x}$, and $V_{x x}$ represent the first-order and secondorder partial derivatives of $V(t, x)$ with respect to the variables $t, x$.

Assume that $H(t, x) \in C^{1,2}([0, T] \times \mathbb{R})$ is a solution of (11); then, according to the first-order condition the optimal investment and consumption strategy is given by

$$
\begin{gathered}
\dot{U}_{1}\left(C^{*}(t)\right)=\frac{H_{x}}{\alpha e^{-\beta t}}, \\
\pi^{*}(t)=-\left(\sigma(t) \sigma^{\prime}(t)\right)^{-1} B(t) \frac{H_{x}}{H_{x x}} .
\end{gathered}
$$

Letting $\theta(t)=\sigma^{-1}(t) B(t)$ and substituting (13) into (11), we obtain

$$
\begin{gathered}
H_{t}+r(t) x H_{x}-\frac{1}{2}\|\theta(t)\|^{2} \frac{H_{x}^{2}}{H_{x x}}-C^{*}(t) H_{x} \\
+\alpha e^{-\beta t} U_{1}\left(C^{*}(t)\right)=0,
\end{gathered}
$$

with boundary condition given by $H(T, x)=(1-$ $\alpha) e^{-\beta T} U_{2}(x)$.
For HARA utility, it is very difficult to directly conjecture the form of the solution of (14). Therefore, we introduce the following Legendre transform technique.

Definition 2. Let $f: \mathbb{R}^{n} \rightarrow \mathbb{R}$ be a convex function. Legendre transform can be defined as follows:

$$
L(z)=\max _{x}\{f(x)-z x\}
$$

and then the function $L(z)$ is called the Legendre dual function of $f(x)$ (cf. Jonsson and Sircar [10], Xiao et al. [11], and Gao $[12,13])$.

If $f(x)$ is strictly convex, the maximum in (15) will be attained at just one point, which we denoted by $x_{0}$. We can attain at the unique solution by the first-order condition:

$$
\frac{d f(x)}{d x}-z=0
$$

So we have

$$
L(z)=f\left(x_{0}\right)-z x_{0} .
$$

Following Jonsson and Sircar [10], Xiao et al. [11], and Gao $[12,13]$, Legendre transform can be defined by

$$
\widehat{H}(t, z)=\sup _{x>0}\{H(t, x)-z x\},
$$

where $z>0$ denotes the dual variable to $x$. The value of $x$ where this optimum is attained is denoted by $g(t, z)$; so we have

$$
g(t, z)=\inf _{x>0}\{x \mid H(t, x) \geqslant z x+\widehat{H}(t, z)\} .
$$

The relationship between $\widehat{H}(t, z)$ and $g(t, z)$ is given by

$$
g(t, z)=-\widehat{H}_{z}
$$

Hence, we can choose either one of two functions $g(t, z)$ and $\widehat{H}(t, z)$ as the dual function of $H(t, x)$. In this paper we choose $g(t, z)$. Moreover, we have

$$
H_{x}=z, \quad \widehat{H}(t, z)=H(t, g)-z g, \quad g(t, z)=x .
$$

Differentiating (21) with respect to $t$ and $z$, we get

$$
H_{t}=\widehat{H}_{t}, \quad H_{x}=z, \quad H_{x x}=-\frac{1}{\widehat{H}_{z z}} .
$$

Notice that $H(T, x)=(1-\alpha) e^{-\beta T} U_{2}(x)$; then at the terminal time $T$, we can define

$$
\begin{aligned}
\widehat{H}(T, z) & =\sup _{x>0}\{H(T, x)-z x\}, \\
g(T, z) & =\inf _{x>0}\{x \mid H(T, x) \geqslant z x+\widehat{H}(T, z)\} .
\end{aligned}
$$

So we have $g(T, z)=\dot{U}_{2}^{-1}\left(z /\left((1-\alpha) e^{-\beta T}\right)\right)$, where $\left(\dot{U}_{2}\right)^{-1}(\cdot)$ is taken as the inverse of marginal utility. 
Putting (22) into (14), we obtain

$$
\begin{gathered}
\widehat{H}_{t}+r(t) z g+\frac{1}{2}\|\theta(t)\|^{2} z^{2} \widehat{H}_{z z}-C^{*}(t) z \\
+\alpha e^{-\beta t} U_{1}\left(C^{*}(t)\right)=0 .
\end{gathered}
$$

Differentiating (24) with respect to $z$ and using (20), we derive

$$
\begin{gathered}
g_{t}-r(t) g+\left(\|\theta(t)\|^{2}-r(t)\right) z g_{z}+\frac{1}{2}\|\theta(t)\|^{2} z^{2} g_{z z} \\
+\frac{\partial\left(C^{*}(t) z\right)}{\partial z}-\frac{\partial\left(\alpha e^{-\beta t} U_{1}\left(C^{*}(t)\right)\right)}{\partial z}=0,
\end{gathered}
$$

with boundary condition given by $g(T, z)=\dot{U}_{2}^{-1}(z /((1-$ $\left.\left.\alpha) e^{-\beta T}\right)\right)$.

Under HARA utility, we have

$$
\begin{aligned}
g(T, z)= & \frac{1-p}{q}(1-\alpha)^{-1 /(p-1)}\left(e^{\beta T}\right)^{1 /(p-1)} \\
& \times z^{1 /(p-1)}-\frac{1-p}{q} \eta .
\end{aligned}
$$

\section{The Optimal Investment and Consumption Strategy}

Applying (22) to (13), we get

$$
C^{*}(t)=\frac{1-p}{q} \alpha^{-1 /(p-1)}\left(e^{\beta t}\right)^{1 /(p-1)} z^{1 /(p-1)}-\frac{1-p}{q} \eta .
$$

Assume that a solution $g(t, z)$ of $(25)$ with terminal condition (26) is given by

$$
\begin{aligned}
g(t, z)= & \frac{1-p}{q}(1-\alpha)^{-1 /(p-1)}\left(e^{\beta t}\right)^{1 /(p-1)} z^{1 /(p-1)} D_{1}(t) \\
& -\frac{1-p}{q} \eta D_{2}(t), \quad D_{1}(T)=1, D_{2}(T)=1 .
\end{aligned}
$$

Then the partial derivatives are derived as follows:

$$
\begin{aligned}
g_{t}= & \frac{1-p}{q}(1-\alpha)^{-1 /(p-1)}\left(e^{\beta t}\right)^{1 /(p-1)} z^{1 /(p-1)} \\
& \times\left(\frac{1}{p-1} \beta D_{1}(t)+\dot{D}_{1}(t)\right)-\frac{1-p}{q} \eta \dot{D}_{2}(t), \\
g_{z}= & \frac{1-p}{q} \cdot \frac{1}{p-1}(1-\alpha)^{-1 /(p-1)}\left(e^{\beta t}\right)^{1 /(p-1)} \\
& \times z^{(1 /(p-1))-1} D_{1}(t), \\
g_{z z}= & \frac{1-p}{q} \cdot \frac{2-p}{(p-1)^{2}}(1-\alpha)^{-1 /(p-1)}\left(e^{\beta t}\right)^{1 /(p-1)} \\
& \times z^{(1 /(p-1))-2} D_{1}(t) .
\end{aligned}
$$

Plugging (27)-(31) into (25), we obtain

$$
\begin{gathered}
\frac{1-p}{q}(1-\alpha)^{-1 /(p-1)}\left(e^{\beta t}\right)^{1 /(p-1)} z^{1 /(p-1)} \\
\times\left(\dot{D}_{1}(t)+\left(\frac{p}{2(p-1)^{2}}\|\theta(t)\|^{2}\right.\right. \\
\left.-\frac{p}{p-1} r(t)+\frac{1}{p-1} \beta\right) D_{1}(t) \\
\left.+\left(\frac{\alpha}{1-\alpha}\right)^{-1 /(p-1)}\right) \\
-\frac{1-p}{q} \eta\left(\dot{D}_{2}(t)-r(t) D_{2}(t)+1\right)=0 .
\end{gathered}
$$

Further, (32) can be decomposed into the following two equations:

$$
\begin{aligned}
\dot{D}_{1}(t)+ & \left(\frac{p}{2(p-1)^{2}}\|\theta(t)\|^{2}-\frac{p}{p-1} r(t)+\frac{1}{p-1} \beta\right) D_{1}(t) \\
+ & \left(\frac{\alpha}{1-\alpha}\right)^{-1 /(p-1)}=0, \quad D_{1}(T)=1 ; \\
& \dot{D}_{2}(t)-r(t) D_{2}(t)+1=0, \quad D_{2}(T)=1 .
\end{aligned}
$$

Solving (33), we get

$$
\begin{aligned}
& D_{1}(t)=e^{\int_{t}^{T} a(s) d s}+\left(\frac{\alpha}{1-\alpha}\right)^{-1 /(p-1)} \int_{t}^{T} e^{\int_{t}^{s} a(u) d u} d s, \\
& D_{2}(t)=e^{-\int_{t}^{T} r(s) d s}+\int_{t}^{T} e^{-\int_{t}^{s} r(u) d u} d s
\end{aligned}
$$

where

$$
a(t)=\frac{p}{2(p-1)^{2}}\|\theta(t)\|^{2}-\frac{p}{p-1} r(t)+\frac{1}{p-1} \beta .
$$

Applying (20), (22), (28), and (30), we have

$$
\begin{aligned}
\frac{H_{x}}{H_{x x}} & =-z \widehat{H}_{z z}=z g_{z} \\
& =\frac{1-p}{q} \cdot \frac{1}{p-1}(1-\alpha)^{-1 /(p-1)}\left(e^{\beta t}\right)^{1 /(p-1)} z^{1 /(p-1)} D_{1}(t) \\
& =\frac{1}{p-1}\left(g+\frac{1-p}{q} \eta D_{2}(t)\right) \\
& =\frac{1}{p-1}\left(x+\frac{1-p}{q} \eta D_{2}(t)\right) .
\end{aligned}
$$

Meantime, (27) can be rewritten as

$$
\begin{aligned}
C^{*}(t)= & \left(\frac{\alpha}{1-\alpha}\right)^{-1 /(p-1)}\left(x+\frac{1-p}{q} \eta D_{2}(t)\right) D_{1}^{-1}(t) \\
& -\frac{1-p}{q} \eta .
\end{aligned}
$$


On the other hand, according to $g(t, z)=x$ and (28), we derive

$$
z=(1-\alpha) e^{-\beta t}\left(\left(\frac{q}{1-p} x+\eta D_{2}(t)\right) D_{1}^{-1}(t)\right)^{p-1}
$$

Considering $H_{x}=z$, we obtain the following optimal value function:

$$
\begin{aligned}
H^{*}(t, x)= & (1-\alpha) e^{-\beta t} \frac{1-p}{q p}\left(\frac{q}{1-p} x+\eta D_{2}(t)\right)^{p} \\
& \times\left(D_{1}(t)\right)^{1-p} .
\end{aligned}
$$

Summarizing what is mentioned above and considering $X(t)=x$, we can draw the following conclusions.

Theorem 3. Under HARA utility (6), the optimal investment and consumption strategy of the problem (5) is given by

$$
\begin{aligned}
\pi^{*}(t)= & \frac{1}{1-p}\left(\sigma(t) \sigma^{\prime}(t)\right)^{-1} B(t)\left(X(t)+\frac{1-p}{q} \eta D_{2}(t)\right) \\
C^{*}(t)= & \left(\frac{\alpha}{1-\alpha}\right)^{-1 /(p-1)}\left(X(t)+\frac{1-p}{q} \eta D_{2}(t)\right) D_{1}^{-1}(t) \\
& -\frac{1-p}{q} \eta,
\end{aligned}
$$

where $D_{1}(t)$ and $D_{2}(t)$ are given by (34) and (35), respectively.

Theorem 4 (Verification theorem). Let $H(t, x) \quad \epsilon$ $C^{1,2}([0, T] \times \mathbb{R})$ be a solution to (11); then, for all admissible strategies $(\pi(t), C(t)) \in \Gamma$, we have $V(t, x) \leqslant H(t, x)$; if there exists $\left(\pi^{*}(t), C^{*}(t)\right) \in \Gamma$ satisfies

$$
\left(\pi^{*}(t), C^{*}(t)\right) \in \arg \sup _{(\pi(t), C(t)) \in \Gamma}\{\mathscr{A} H(t, x)\} ;
$$

then we conclude that $V(t, x)=H(t, x)$ and $\left(\pi^{*}(t), C^{*}(t)\right)$ given by Theorem 3 is the optimal investment and consumption strategy of the problem (5).

Proof. Considering $H(t, x) \in C^{1,2}([0, T] \times \mathbb{R})$ and using Itô formula from $t$ to $T$ for $H(t, x)$, we obtain

$$
\begin{aligned}
H(T, X(T))= & H(t, x)+\int_{t}^{T} \mathscr{A}^{\pi, C} H(s, X(s)) d s \\
& +\int_{t}^{T} \pi^{\prime}(s) \sigma(s) H_{x}(s, X(s)) d W(s) .
\end{aligned}
$$

Considering that $H(t, x)$ is a solution to (11); that is, we have $\sup _{(\pi(t), C(t)) \in \Gamma} \mathscr{A}^{\pi, C} H(s, X(s))=0$. So we obtain

$$
\begin{aligned}
& H(T, X(T)) \\
& \quad \leqslant H(t, x)+\int_{t}^{T} \pi^{\prime}(s) \sigma(s) H_{x}(s, X(s)) d W(s) .
\end{aligned}
$$

The last one term in (45) is a local martingale and its expected value is equal to zero. Thus, we get

$$
E(H(T, X(T)) \mid X(t)=x) \leqslant H(t, x) .
$$

Maximizing (46) for all admissible strategies $(\pi(t), C(t)) \in \Gamma$, we derive

$$
V(t, x) \leqslant H(t, x)
$$

When $(\pi(t), C(t))=\left(\pi^{*}(t), C^{*}(t)\right)$, all inequalities become equalities; that is, $H(t, x)=V(t, x)$, and it shows that $\left(\pi^{*}(t), C^{*}(t)\right)$ given by Theorem 3 is the optimal investment and consumption strategy of the problem (5).

The proof is completed.

In particular, we can obtain the following three special cases.

Corollary 5. If $\eta=0$ and $q=1-p$, HARA utility is degenerated to power utility. Therefore, the optimal investment and consumption strategy of the problem (5) under $U_{1}(x)=$ $U_{2}(x)=x^{p} / p$ is given by

$$
\begin{array}{r}
\pi^{*}(t)=\frac{1}{1-p}\left(\sigma(t) \sigma^{\prime}(t)\right)^{-1} B(t) X(t), \\
C^{*}(t)=\left(\int_{t}^{T}\left(\frac{\alpha}{1-\alpha}\right)^{1 /(p-1)} e^{\int_{t}^{T} a(s) d s}\right. \\
\left.+\int_{t}^{T} e^{\int_{t}^{s} a(u) d u} d s\right)^{-1} X(t),
\end{array}
$$

where $a(t)$ is given by (36).

Corollary 6. If $\eta=1$ and $p \rightarrow-\infty, H A R A$ utility is reduced to exponential utility. Therefore, the optimal investment and consumption strategy of the problem (5) under $U_{1}(x)=$ $U_{2}(x)=-e^{-q x} / q$ is given by

$$
\pi^{*}(t)=\frac{1}{q}\left(\sigma(t) \sigma^{\prime}(t)\right)^{-1} B(t) D_{2}(t),
$$

$$
\begin{aligned}
C^{*}(t)= & D_{2}^{-1}(t) X(t) \\
& +\frac{1}{q} D_{2}^{-1}(t)\left(e^{-\int_{t}^{T} r(z) d z} \cdot \ln \frac{\alpha}{1-\alpha}\right. \\
& +e^{-\int_{t}^{T} r(z) d z} \cdot \int_{t}^{T} \tilde{a}(s) d s \\
& \left.+\int_{t}^{T}\left(e^{-\int_{t}^{s} r(z) d z} \int_{t}^{s} \tilde{a}(z) d z\right) d s\right),
\end{aligned}
$$

where $D_{2}(t)$ is given by (35), and $\widetilde{a}(t)$ is given by

$$
\tilde{a}(t)=\frac{1}{2}\|\theta(t)\|^{2}+\beta-r(t)
$$


Proof. The equation (41) in Theorem 3 can be rewritten as

$$
\begin{aligned}
\pi^{*}(t)= & \frac{1}{1-p}\left(\sigma(t) \sigma^{\prime}(t)\right)^{-1} B(t) X(t) \\
& +\frac{1}{q} \eta D_{2}(t)\left(\sigma(t) \sigma^{\prime}(t)\right)^{-1} B(t) .
\end{aligned}
$$

When $\eta=1$ and $p \rightarrow-\infty$, then we have

$$
\pi^{*}(t)=\frac{1}{q}\left(\sigma(t) \sigma^{\prime}(t)\right)^{-1} B(t) D_{2}(t) .
$$

Therefore, it is obvious that (49) is proved.

Similarly, (42) can be changed into

$$
\begin{aligned}
C^{*}(t)= & \left(\frac{\alpha}{1-\alpha}\right)^{-1 /(p-1)} D_{1}^{-1}(t) X(t) \\
& +\frac{1-p}{q} \eta\left(D_{2}(t)\left(\frac{\alpha}{1-\alpha}\right)^{-1 /(p-1)} D_{1}^{-1}(t)-1\right) .
\end{aligned}
$$

When $p \rightarrow-\infty$, we have $a(t) \rightarrow-r(t)$ and $D_{1}(t) \rightarrow D_{2}(t)$. Therefore, it leads to that

$$
\lim _{p \rightarrow-\infty}\left(\frac{\alpha}{1-\alpha}\right)^{-1 /(p-1)} D_{1}^{-1}(t) X(t)=D_{2}^{-1}(t) X(t) .
$$

For the second term in (54), we get

$$
\begin{aligned}
& \lim _{p \rightarrow-\infty} \frac{1-p}{q} \eta\left(D_{2}(t)\left(\frac{\alpha}{1-\alpha}\right)^{-1 /(p-1)} D_{1}^{-1}(t)-1\right), \\
& \quad=\frac{1}{q} \lim _{p \rightarrow-\infty} \frac{D_{2}(t)(\alpha /(1-\alpha))^{-1 /(p-1)} D_{1}^{-1}(t)-1}{1 /(p-1)}, \quad\left(\frac{0}{0}\right) .
\end{aligned}
$$

By using L'Hopital's rule, (56) is equal to

$$
\begin{gathered}
\frac{1}{q} D_{2}^{-1}(t)\left(e^{-\int_{t}^{T} r(z) d z} \cdot \ln \frac{\alpha}{1-\alpha}+e^{-\int_{t}^{T} r(z) d z} \cdot \int_{t}^{T} \tilde{a}(s) d s\right. \\
\left.+\int_{t}^{T}\left(e^{-\int_{t}^{s} r(z) d z} \int_{t}^{s} \tilde{a}(z) d z\right) d s\right) .
\end{gathered}
$$

As a result, (50) is also proved.

Corollary 7. If $\eta=0, p \rightarrow 0$, and $q \rightarrow 1$, HARA utility is reduced to logarithm utility. It leads to $a(t)=-\beta$. Therefore, the optimal investment and consumption strategy of the problem (5) under $U_{1}(x)=U_{2}(x)=\ln x$ is given by

$$
\begin{aligned}
\pi^{*}(t) & =\left(\sigma(t) \sigma^{\prime}(t)\right)^{-1} B(t) X(t) \\
C^{*}(t) & =\frac{\alpha}{(1-\alpha) e^{-\beta(T-t)}-(\alpha / \beta)\left(e^{-\beta(T-t)}-1\right)} X(t) .
\end{aligned}
$$

\section{Conclusions}

This paper is concerned with a class of investment and consumption problems with HARA utility. We present a Legendre transform method to deal with them and obtain the explicit expressions of the optimal investment and consumption strategy. Some special cases are also discussed.

\section{Conflict of Interests}

The authors declare that there is no conflict of interests regarding the publication of this paper.

\section{Acknowledgments}

This research is supported by National Natural Science Foundation of China (no. 11301376), Humanities and Social Science Research Youth Foundation of Ministry of Education of China (no. 11YJC790006), and Higher School Science and Technology Development Foundation of Tianjin (no. 20100821).

\section{References}

[1] R. C. Merton, "Lifetime portfolio selection under uncertainty: the continuous-time case," The Review of Economics and Statistics, vol. 51, no. 3, pp. 247-257, 1969.

[2] R. C. Merton, "Optimum consumption and portfolio rules in a continuous-time model," Journal of Economic Theory, vol. 3, no. 4, pp. 373-413, 1971.

[3] G. Chacko and L. M. Viceira, "Dynamic consumption and portfolio choice with stochastic volatility in incomplete markets," Review of Financial Studies, vol. 18, no. 4, pp. 1369-1402, 2005.

[4] J. Liu, "Portfolio selection in stochastic environments," Review of Financial Studies, vol. 20, no. 1, pp. 1-39, 2007.

[5] E.-J. Noh and J.-H. Kim, "An optimal portfolio model with stochastic volatility and stochastic interest rate," Journal of Mathematical Analysis and Applications, vol. 375, no. 2, pp. 510$522,2011$.

[6] H. Chang and X.-M. Rong, "An investment and consumption problem with CIR interest rate and stochastic volatility," Abstract and Applied Analysis, vol. 2013, Article ID 219397, 12 pages, 2013.

[7] H. Chang, X. M. Rong, H. Zhao, and C. B. Zhang, "Optimal investment and consumption decisions under the constant elasticity of variance model," Mathematical Problems in Engineering, vol. 2013, Article ID 974098, 11 pages, 2013.

[8] M. Grasselli, "A stability result for the HARA class with stochastic interest rates," Insurance: Mathematics and Economics, vol. 33, no. 3, pp. 611-627, 2003.

[9] E. J. Jung and J. H. Kim, "Optimal investment strategies for the HARA utility under the constant elasticity of variance model," Insurance: Mathematics and Economics, vol. 51, no. 3, pp. 667673, 2012.

[10] M. Jonsson and R. Sircar, "Optimal investment problems and volatility homogenization approximat ions," in Modern Methods in Scientific Computing and Applications, vol. 75 of NATO Science Series II, pp. 255-281, Springer, Berlin, Germany, 2002.

[11] J. Xiao, Z. Hong, and C. Qin, “The constant elasticity of variance (CEV) model and the Legendre transform-dual solution for 
annuity contracts," Insurance: Mathematics and Economics, vol. 40, no. 2, pp. 302-310, 2007.

[12] J. Gao, "Optimal investment strategy for annuity contracts under the constant elasticity of variance (CEV) model," Insurance: Mathematics and Economics, vol. 45, no. 1, pp. 9-18, 2009.

[13] J. Gao, "An extended CEV model and the Legendre transformdual-asymptotic solutions for annuity contracts," Insurance: Mathematics and Economics, vol. 46, no. 3, pp. 511-530, 2010.

[14] W. H. Fleming and H. M. Soner, Controlled Markov Processes and Viscosity Solutions, Springer, New York, NY, USA, 2nd edition, 2006. 


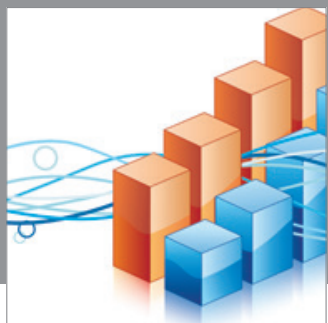

Advances in

Operations Research

mansans

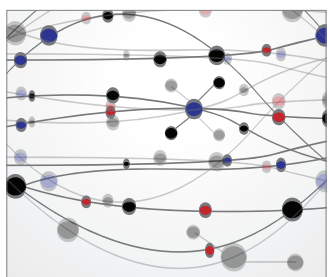

The Scientific World Journal
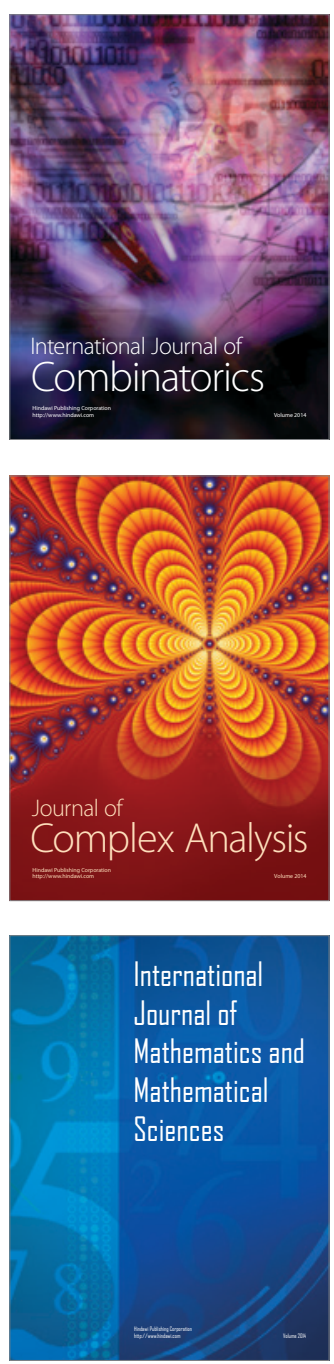
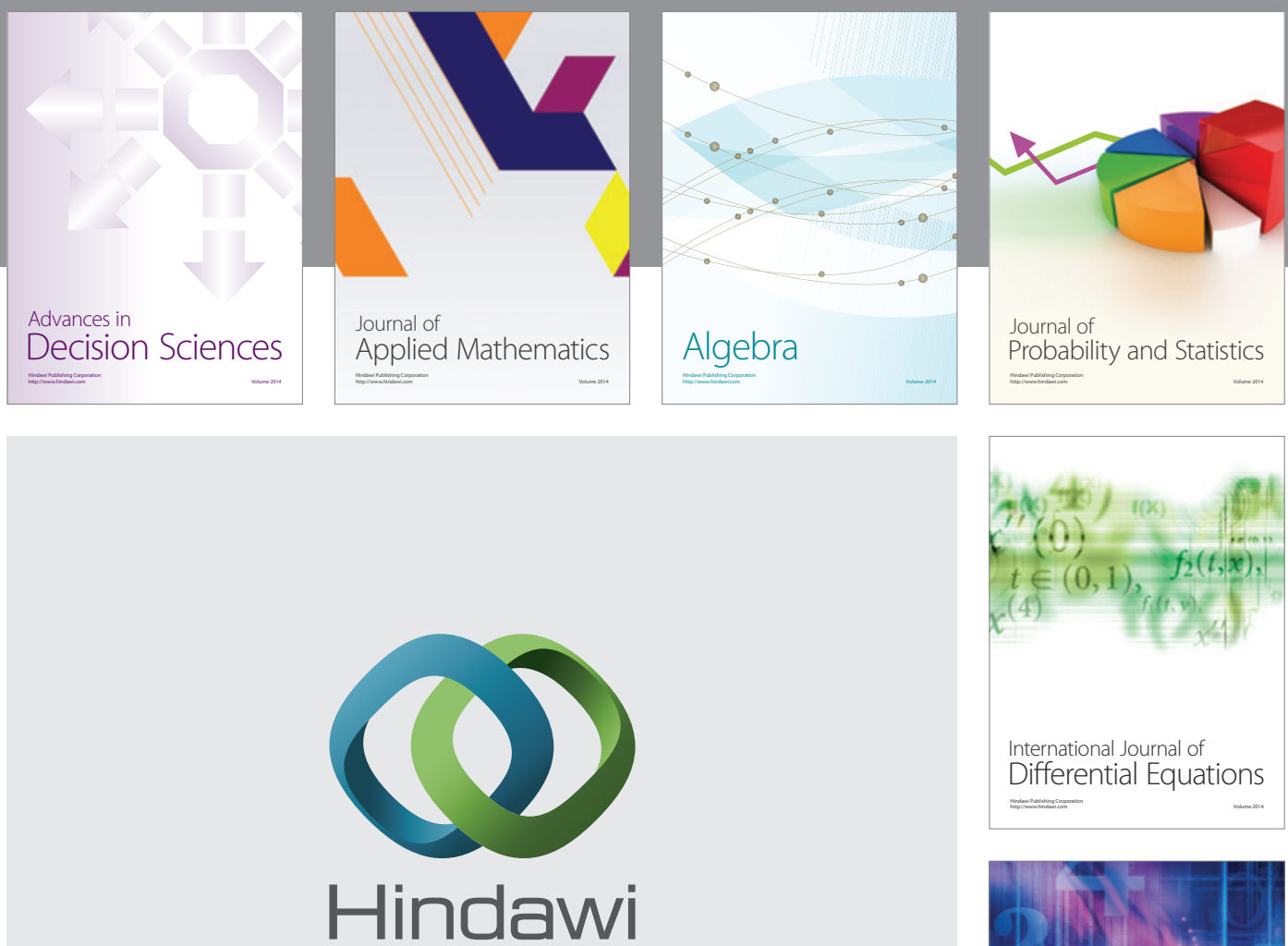

Submit your manuscripts at http://www.hindawi.com
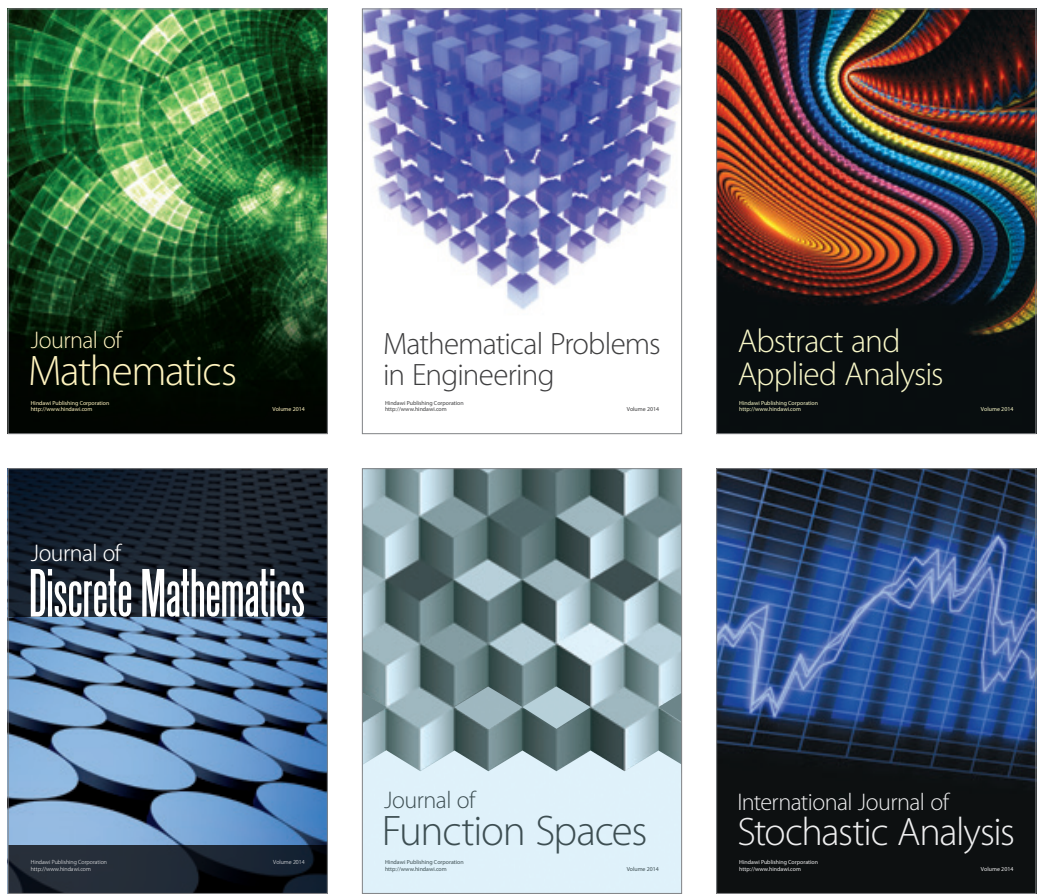

Journal of

Function Spaces

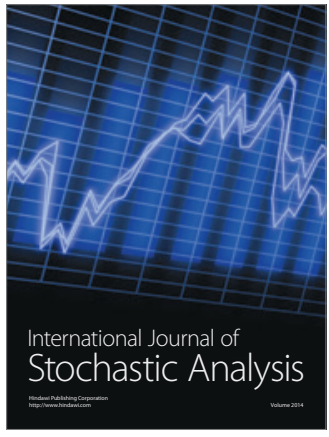

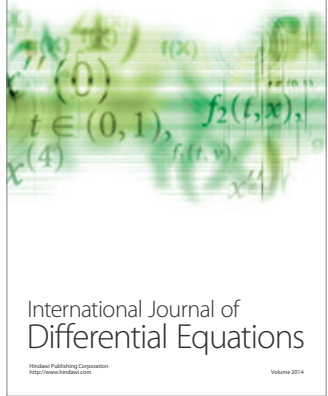
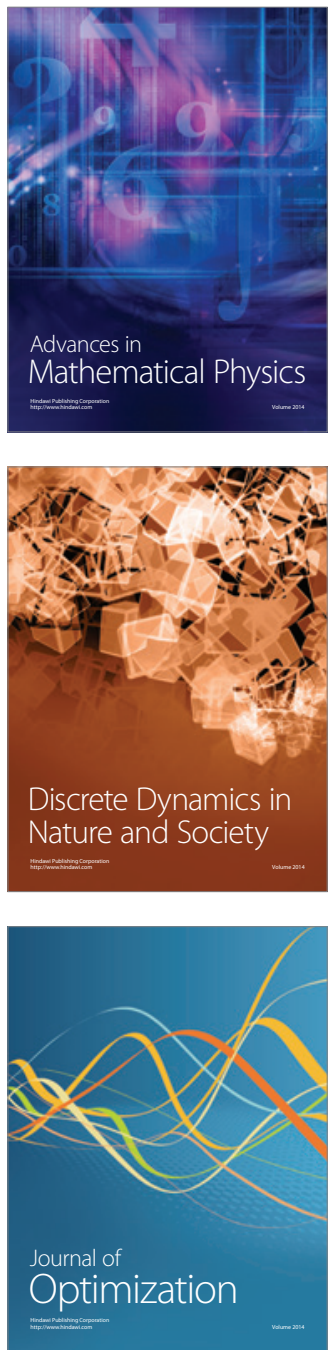